\title{
Model Fusion at the British Geological Survey - experiences and future trends
}

Denis Peach, Andrew Riddick, Andrew Hughes, Holger Kessler, Steve Mathers, Christopher Jackson and Jeremy Giles

\section{Abstract}

The British Geological Survey (BGS) is developing integrated environmental models to address the grand challenges that face society. Here we describe the BGS vision for an Environmental Modelling Platform (BGS 2009), that will allow integrated models to be built and describe case studies of emerging models in the United Kingdom.

This Environmental Modelling Platform will be founded on the data and information that BGS holds. This will have to be made as accessible and interoperable as possible to both the academic and stakeholder decision making community. The geological models that have been built in an adhoc way over the last 5-10 years will be encompassed in a National Geological Model which will be multi-scaled, beginning with onshore United Kingdom and eventually including the offshore continental shelf. The future will be characterised by the routine delivery of 3D model products from a multi-scaled and scalable 3D geological model of the UK which can be dynamically updated. The deployment of this model will generate further significant requirements across the Information and Knowledge Exchange spectrum, from applications development (database, GIS, web and mobile device), data management, information product development, to delivery to a growing number of publics and stakeholders.

There is now a growing realisation in the environmental and social sciences that to address the grand challenges that face the world a whole system approach is required. These challenges including climate change, natural resource and energy security and environment vulnerability raise multi- and transdisciplinary issues that require integrated understanding and analysis. Not only must we model the whole physical Earth system, bringing together climate, ecological, hydrological, hydrogeological, and geological models to name but a few, we must link them to socio- economic models. Model fusion may well be the only adequate way to provide the necessary coupled processes framework through which predictions and planning or management decisions can most appropriately be made.

A scoping study (Giles et al 2010) assessed the current situation and made some preliminary recommendations in order to create a more integrated and semantically harmonized future in environmental modelling. The only viable option is a linked models' approach which enables models to pass parameters between each other at runtime. This solution can bring together the best and most appropriate scientific models and allows the various scientific disciplines to continue the development of their current models. This linking approach is also relevant for integrating models that have been largely built and optimised individually, with appropriate configuration. The European Union has funded multi-national, multi-disciplinary research into 'linked modelling', using the Open Model Interchange (OpenMI) standard. This software used, in conjunction with critical underpinning activities such as data management, semantics and ontologies, understanding of uncertainty and visualisation, offers a rapidly maturing solution, the creation of an Environmental Modelling Platform, with the potential to fulfil this vision. 
The British Geological Survey has a long history of developing 3D geological framework models as well as groundwater flow models and other process models like those to assess impacts of carbon storage in the subsurface. Increasingly the survey is now producing $3 \mathrm{D}$ geological models alongside or as an alternative to $2 \mathrm{D}$ geological maps. It is clear that there has been a proliferation of modelling activities across many other environmental science disciplines. Thus a significant number of environmental models are now available, including models to predict environmental hazards and habitat quality and those focussed on environmental resource sustainability whilst such models represent significant advances in technology, and frequently considerable investments in time and intellectual effort, they are often discipline specific and developed to address a specific issue or problem. The ability to link such models together allows a holistic understanding of earth system processes, . These linkages and the systems-level understanding that they provide are increasingly important in helping us to solve overarching environmental problems facing the world today such as (1)predicting and responding to environmental change, (2) ensuring the security of natural resources for the future, and ( 3 ) understanding and predicting natural hazards. It is only through harnessing some of the existing large and very complex models available that further progress can be made in addressing these issues.

Specific examples of the application of linked and integrated modelling could include linking groundwater models with models predicting climate and future land-use trends in order to better understand the impact of climate change on agricultural policies and planning regulations. In such a scenario BGS clearly has an important role in contributing our knowledge of the 3D distribution of rock units, groundwater movement, and the impact of geology on land-use. The integration of large scale climate models with possibly more localised models of groundwater, rainfall and landuse brings us to the importance of being able to deal with heterogeneity and scale between different models. Understanding the interaction between groundwater levels and changes in sea level due to climate change is also a topical issue in a number of parts of the UK, and requires fusing together groundwater, rainfall, and other climate models. There are also numerous instances in the sub-surface where being able understand the interaction between different flow regimes is critical, for example in planning carbon dioxide sequestration.

In addition to drivers from the wider research community, the BGS strategy requires the interpretation and analysis of a range of environmental and resource observations in an integrated way. In order to accomplish this we are aware of an increasing need to be able to integrate different models and datasets to answer science questions, and to provide the outputs from these modelling activities to users in a form which they can use directly. This objective involves creating both the IT infrastructure components to support this work, and developing the organisational culture and ways of working needed. The importance of addressing the requirements of a number of stakeholders(not only the modelling specialist creating the model) in the modelling process, particularly in appreciating the inputs to a model and the outputs from it has also been pointed out by Voinov et al. (2016) and Glynn (2016, this volume).The proposed solution is the creation of an open environmental modelling platform which will provide the methodologies, software tools and standards upon which to undertake integrated multi-scaled environmental modelling, providing a sound and reproducible basis for decision making. 


\section{Building the Environmental Modelling Platform}

In order to design the environmental modelling platform BGS undertook a scoping study to understand the future requirements for integrated modelling both within the organisation and more widely. One of the key recommendations of this study was that since many environmental organisations have already invested significant time, cost and effort in developing their models then the focus for onward development should be to harness this investment, rather than to try to propose an entirely new software system. Thus the BGS vision for the environmental modelling platform consists of a portfolio of methodologies, software tools and standards to allow us to model the environment in an integrated way, and to link with relevant models outside the geoscience domain.

The key components within the environmental modelling platform include BGS's data and information resources, as well as existing geological models, conceptual models and process models (for example for groundwater modelling). It can be thought of as the entire research and knowledge base of the BGS. Figure 1 shows the conceptual relationship between these components. Our data and information resources form the foundation for the platform represented by the base of the triangle in this figure. 3D geological framework models are then built using this input data, and forms one of a number of means of constraining conceptual models and process models. Progressing from 3D geological framework modelling through the development of a conceptual model, and then the process modelling itself allows predictive modelling and scenario planning.

There are a number of challenges to be overcome to construct the environmental modelling platform. These include issues concerning underpinning technologies, challenges in developing appropriate modelling methods, for example for model parameterisation and uncertainty quantification. There are also challenges in developing the required workflows and cultural practices both within the organisation and externally and in the delivery of products.

The main challenges and under-pinning areas of work are discussed below:

\section{Standards}

There are a wide variety of software data and modelling standards that are applicable to environmental modelling. BGS's approach is to support and adopt existing standards wherever possible, and to create new standards only where this is absolutely necessary. The OpenMI methodology for linking models at run-time has been described above, and this has now been adopted as an Open Geospatial Consortium (OGC) standard (OGC 2013). The drivers for adoption of the Open MI as an OGC standard included an interest by the OGC in addressing the time dimension, and also the value of the OpenMI in the increasing efforts to link sensors, datasets, and models within the OGC's community of users. The OpenMI Standard can be implemented using a software 
development toolkit downloadable from the OpenMI Association website, or alternatively via open source tools also downloadable from the web, or users can write their own code using information provided by the OpenMI Association. The use of these tools and implementation methods is optional and does not form part of the standard. .

Other standards employed by BGS in developing integrated modelling include GeoSciML for representing data vocabularies (Sen and Duffy 2005), and the ISO metadata standards (ISO 19115-1, 2014) for data quality and discovery metadata.

\section{Software}

A number of methodologies to link environmental models particularly at run-time have been proposed. One approach is offered by the Open Modelling Interface (OpenMI) Association (Gregerson et al, 2007) which has produced an open standard for exchanging information between OpenMI compliant models at run-time. A demonstration project, financed by the European Commission - Life Programme, was centred on the transnational Scheldt River Basin. Water management in the basin is distributed among many different authorities and operators in three countries; Belgium, France and Netherlands. The introduction of the European Water Framework Directive requires water management to be integrated. Existing models have been developed independently, so that integration is far from straightforward. The OpenMI Standard has provided a mechanism to enable the existing models to work together. Important features of Open $\mathrm{MI}$ are that is allows parameters to be passed between models at run-time, it is also implemented as a software "wrapper" around modelling code developed for example in languages such as $\mathrm{C}++$ and therefore permits ready reuse of code.

Gregerson et al. (2005 and 2007) describe how OpenMI provides a standardized interface to define, describe and transfer data on a time basis between software components that run simultaneously. This supports systems where feedback between the modelled processes is necessary in order to achieve physically sound results. The way the OpenMI works is by modifying models into three distinct parts: initialise, run and finalise. The initialise part sets up the model, reads in data and so on, the run part allows the control of one time step at a time and the finalise part then "tidies" up the model at the end of the run. This could mean writing out the results, releasing memory amongst other things. (see Figure 2). The model with the smallest time step controls the simulation. It marches forward one time step and then requests data from the model components linked to it (Figure 3). This model ("component B") then interpolates the value and returns it to model component $A$. This is repeated until the end of model component B's time step at which a "real" value is returned. This whole process is repeated until the simulation is finished.

In this way the OpenMI allows the linking of models with different spatial and temporal representations; for example, linking river models and groundwater models, where the river model typically uses a one-dimensional grid and a short time step, and the 
groundwater model uses a 2D or 3D grid and a longer time step. The OpenMI method has now been applied extensively within BGS particularly for work on groundwater and climate impact modelling

\section{Semantic concepts and the use of ontologies}

The capability of software to integrate models depends to a great extent upon how relationships between important underlying concepts are communicated between models by the software. There is a requirement for such concepts to be communicated in both a human understandable form to facilitate model design and also in machine readable form.

The Semantic Web (Berners-Lee et. al. 2001) uses standard formats to integrate and combine resources from diverse sources. This allows a person, or a machine, to follow a trail of links through multiple data sources. One of the results of this is to enable natural language querying through establishing unique resource identifiers, a standard way to describe resources (resource description format - RDF), and the use of ontologies. An ontology is a structured way of representing real world concepts. Semantics enable us to exchange information and knowledge about an object or concept that exists in an ontology. BGS has been developing a set of standardised database dictionaries which represent vocabularies of key geological terms and concepts as a basis for such ontology development. A key onward development will be to establish relationships and equivalences between geological vocabularies and those used in other environmental science disciplines. This is actually less of a technical task and more of a community building challenge in bringing relevant researchers together to discuss the concepts involved.

\section{Data Management and Quality}

Well managed datasets are those that are easily accessible, contain timely data, and are stored in a secure environment. In general scientists spend considerable amounts of time searching for and formatting datasets so that they are usable. Well managed datasets are said to be accessible when the dataset is easy to locate and retrieve from a data store, they are available in the format in which they are normally used and the intellectual property rights are clearly understood and articulated.

BGS has directed significant resources over recent years to create a well-documented data architecture which supports environmental modelling at a range of scales. Our borehole and geological property datasets form a key element of this, as does the adoption of internationally recognised metadata standards. We have also created a range of applications and tools to permit browsing and searching our data sets in a manner which fits with the workflow of geological and environmental modellers.

Specifically we are developing a database storage system to store our 2D and 3D data used in modelling. This is currently in progress and is discussed in more detail below. We are also involved in a management process to improve data quality following the NERC Science Information Strategy (2009).

\section{Modelling scale heterogeneity and uncertainty}


In the development of multi-scaled models of various types, attributed with sub-surface properties there are significant challenges to be overcome. In order, for example, to develop a 3D geological model of the UK, based on the integration of a large number of regional models, it is necessary to integrate models at different scales. In addition to constructing the framework model providing decision making functionality requires that the models can be attributed with parameters to represent the physical properties of the subsurface e.g. geotechnical properties, porosity, permeability etc. There is also a considerable difficulty in delivering models to users (often outside the BGS) in a form in which they can be easily used. Clearly many users do not have access to the specialist modelling software used within BGS, and so delivery mechanisms need to take this into account. Delivery of models must also be built with an understanding of the specific requirements of the user community. Perhaps the most critical issue to be addressed in model delivery is how to communicate the inevitable uncertainties embodied within a multi-dimensional model.

BGS is addressing the issues surrounding integrating models at a range of scales through the development of a National 3D Geological Model of the UK, built upon various regional models. Parameterisation of models and the uncertainty and delivery problems are being solved through a number of test-bed implementations of the environmental modelling platform which are discussed in more detail below.

\section{Building a research community}

The technical under-pinning work to enable many of the issues described above is well established or at least underway (e.g. software to support model coupling, and in ensuring sound management and organisation of the input data for modelling). One of the critical factors in enabling integrated environmental modelling to progress further is more cultural in nature. There is a great need to facilitate the creation of a networked and linked research community tackling these issues across various environmental science disciplines, so that the barriers between disciplines can be crossed. This research community should include end users and other stakeholders, not only researchers (Voinov et al 2016, Glynn 2016, this volume)

\section{Progress and implementations}

In order to progress the environmental modelling platform we have sought to base development on several key larger projects which need to include integrated modelling approaches. Two projects in particular are described here: an integrated model of the groundwater systems in the Thames basin in the south east of the UK, and a programme of integrated modelling in the Glasgow area of Scotland, also investigating the interactions between hydrology and geology. Both of these research projects involve the linking together of environmental process models within the framework of the BGS National Geological Model. 
Thames Integrated Model

BGS's Thames integrated model (TIM) links the detailed geology of the Thames catchment with groundwater and surface water hydrology, including rainfall, runoff, and recharge, and to a limited extent the source and resource management applied in the Thames Catchment. There has been particular emphasis on the geology of the greater London area. The project brings together a unique combination of geological, hydrogeological, environmental and socio-economic challenges that are intrinsically linked and impacted by climate change. To address these challenges requires fully attributed 3D models that incorporate information and processes from all of these disciplines so that accurate representations, simulations, forecasts and predictions can be made. These forecasts and "predictions" are required to enable informed decision making and planning for sustainability. Within this project integrated modelling is being applied to understand the interaction between rainfall evaporation and runoff, together with river flows for the River Thames and its tributaries, and groundwater flow.

The importance of interaction between the surface water system and abstraction has been demonstrated by using the TIM linked model composition developed by Mansour et al. (2013). A model composition which allows an appropriate representation of the hydrological system has been developed and is shown diagrammatically Figure 4). Two groundwater models have been developed: one of the Chalk using ZOOMQ3D (Jackson and Spink, 2004) and another of the Jurassic limestones using BGSGW (Mansour et al., 2013). The former is a distributed groundwater flow model and the latter is a semidistributed lumped parameter model that can be used to simulate groundwater behaviour. The boundaries of both models are shown on Figure 4 and it should be noted that for the Chalk model these extend outside of the Thames Basin. This is to ensure that sensible groundwater flow boundaries are defined so as not to erroneously calculate baseflow to rivers whose surface catchments are different to groundwater catchments and unduly affect the impacts of groundwater abstractions.

The two groundwater models are linked via a river model (MCRouter) developed using the Muskingham-Cunge approach (Chadwick and Morfett, 1986). A simple hydraulic river model was chosen to ensure the composition can be tested before including a more complex river model. These three models are driven by run-off and recharge generated by the recharge model ZOODRM (Mansour and Hughes, 2004; Hughes et al., 2008). The run-off is routed to the river model whilst the recharge is passed to the two groundwater flow models (Figure 5). As described above, the Jurassic limestones and Chalk aquifer are not linked via the sub-surface, only via interaction with the River Thames and its tributaries. The two groundwater models and the river model are, therefore, dynamically linked by the model linkage standard OpenMI (Gregersen et al., 2007). This linkage has been facilitated by using the Fluid Earth software development toolkit (SDK) and the associated editor, Pipistrelle to construct compositions (Harpham et al., 2014; Figure 7b). 
The model composition has been applied to a hypothetical situation to test the applicability of the models and what benefit is accrued from linking them. A hypothetical groundwater abstraction of $150 \mathrm{Mlday}^{-1}$ has been used to investigate the impacts of groundwater abstraction on the flows in the River Thames. Thegroundwater abstraction was simulated from a point on the banks of the River Thames (labelled BH on Figure 4). This abstraction was linked to riverflow as measured at a downstream gauging station (labelled GS-B on Figure 4). To investigate the effect of different management regimes two scenarios were simulated: One with a fixed groundwater abstraction of $150 \mathrm{Ml} / \mathrm{d}$ and the other with an abstraction that varies from 50 to 150 $\mathrm{Ml} / \mathrm{d}$ depending on river flow at GS-B (Figure 4). The relationship between river flow and magnitude of groundwater withdrawal was achieved by the inclusion of an abstraction management component in the composition which modified groundwater abstraction during runtime. This component related groundwater abstraction by a simple rule and was included in the composition and linked using OpenMI. The abstracted water is returned to the river some $35 \mathrm{~km}$ upstream (labelled GS-A on Figure 4) of the groundwater abstraction as this represents typical water use, from abstraction, supply to the city of Oxford and after use, discharge to the sewerage system and hence finally sewage effluent returned to the river.

The model composition was simulated for a 30 year period and groundwater heads and the river hydrograph downstream of the groundwater abstractions plotted (see Figure 3 and 4 in Mansour et al., 2013). During conditions of low flow, i.e. during the 1975/6 drought the model simulation resulted in a river flow that was lower for the scenario with fixed abstraction than for the scenario where groundwater abstraction decreased. However, as groundwater abstraction was reduced, groundwater levels surrounding the abstraction were higher. Whilst the variable abstraction reduced the impact on groundwater heads the overall impact on river flows increased due to the reduction in return flows to the River Thames. Whilst this is a hypothetical example, the results are contrary to expectation and the utility of the modelling composition in the development of management policies for droughts, and its potential for other scenarios was demonstrated.

\section{Glasgow Model}

The availability of detailed three-dimensional (3D) geological framework models for the Glasgow area (Merritt et al., 2007) provides an accurate representation of the subsurface within which to evaluate linked groundwater models of the complex Quaternary deposits of the Glasgow area. This study evaluated the use of 3D modelling to constrain groundwater flow predictions building on earlier work in Glasgow which used interpretations of the geology based on 2D maps. An understanding of the 3D geometry of the lithostratigraphic units has allowed a very detailed conceptualisation of the likely groundwater regime. Recharge (Mansour et al., 2008) and groundwater flow models (Turner et al., 2015) have been developed to test this conceptual understanding.

The results from numerical modelling indicate that the general direction of groundwater flow in Glasgow is down-gradient from areas of high ground towards the 
lowland valleys and the River Clyde itself, through both bedrock and Quaternary potential aquifers (Turner et al., 2015). Groundwater levels in the Quaternary Clyde Valley Aquifer are strongly influenced by the course of the River Clyde and its tributaries. The sand deposits within the Quaternary act as a highly conductive shallow aquifer in the Clyde valley, and are responsible for regional flow of shallow groundwater. The model also suggests that Quaternary deposits in the Proto valley of a tributary of the river Clyde constitute a significant aquifer receiving and promoting groundwater to flow towards the River Clyde, but the dearth of local data makes any conclusions conjectural. The conceptual model developed using the 3D modelling an input, has been broadly validated by the numerical modelling. Thus this study illustrates the value of integrating 3D geological framework models with groundwater flow modelling.

The methodologies and modelling frameworks established for the Glasgow area (Figure 6) provide a good basis for further modelling using for example long-term groundwater observation data, which will soon be available from the monitoring network being set up by the British Geological Survey, and the Scottish Environmental Protection Agency (SEPA). In addition to validating the conceptual groundwater model the numerical model also provides an opportunity to simulate possible future scenarios and investigate specific urban development issues and proposals in Glasgow(for example to understand the groundwater processes that may impact the remediation of contaminated land).

\section{The future of integrated modelling at BGS}

Future developments in integrated modelling at BGS are planned along several lines, including further research on parameterising models with appropriate property values, and the development of voxelated models in order to more accurately predict the distribution of chemical and physical properties within the sub-surface. We are also aware of the need to establish a dedicated database of 3D and 4D data to provide storage for these models, support model fusion activities, and to facilitate the delivery of 3D and 4D data to end users. Significant work is also in progress in developing techniques for the visualisation and delivery of 3D/4D data. These developments are discussed in more detail below:

The facility to store 3D and increasingly 4D models in a manner which allows easy retrieval is an important component of the environmental modelling platform. In developing a modelling workflow it is imperative that modellers are able to draw on a data store of previous models, and also incorporate new data which has been assimilated since the last modelling of a particular geographic area. The capacity to be able to validate and sometimes update the model source data in the light of modelling results is also an important requirement. The corporate model data store also needs to be software independent so that it can be accessed by modellers working with a variety of modelling software tools, and allow the exchange of data in a variety of formats. An important requirement arising out of these model fusion activities is the increasing need to be able to store potentially large volumes of time series data in an easily 
accessible manner, and also store multiple versions of models corresponding to different model scenarios, with appropriate metadata.

In addition to the requirements imposed by the modelling workflow, a geological survey organisation also needs to be able to deliver models in a variety of formats and therefore the BGS data store architecture must support this. These key requirements arising from modern modelling best practice are currently guiding the creation of a fully functional database and metadata system to support our modelling activities. This work is building on our experience gained in creating systems to store and access models in the Digital Geospatial Model Project (DGSM) undertaken between 2000 and 2005 (Smith 2005).

The development of mechanisms for model delivery is also an important continuing focus. As mentioned above, models need to be delivered in a format which is designed to meet the user requirements. BGS has maintained a strategy to exploit rapidly developing access to mobile technologies for delivery of a number of our datasets, as exemplified by the BGS iGeology and iGeology3D mobile apps for iPhone and Android platforms. Currently the "Groundhog" application available via the BGS website (http://www.bgs.ac.uk/research/environmentalModelling/groundhogDesktop.html) and for mobile devices provides virtual borehole cross section and horizontal section viewing functionality; Groundhog is a forerunner for further developments for delivery of 3D and 4D data.

In addition to technical developments the importance of close cooperation and collaboration between researchers in different environmental science disciplines has been discussed above, and this continues to be an important area for onward development in BGS. The need to integrate various models in order to address a particular problem has been an important factor in influencing the BGS working culture. We are increasingly creating 3D framework and process models as a standard geological survey output rather than 2D maps, and the need to work in a more integrated way is increasingly reflected in BGS's internal team organisation.

A Natural Environment Research Council Strategy for integrated environmental modelling (IEM) has been adopted by the NERC Research Centres and is currently being refined (Royse and Hughes 2014). The NERC IEM strategy addresses the challenges of linking environmental models to solve specific science questions by creating a community that prioritises modelling needs and a modelling framework to facilitate greater sharing and linking of data and models. The overall objective is to promote a change in culture towards greater collaborative working, improved accessibility and effective use of existing models and tools developed over many years and also to encourage a move away from 'silo management' where each problem is addressed in isolation.

This strategy will address a number of key challenges in IEM including:

- Establishing workable standards that define model input and output parameters and provide a description of the model and the assumptions it is based on. 
- Understanding and quantifying uncertainty, to understand how uncertainty is propagated within a linked modelling system and adopt user specific communication methods.

- Understanding the impact of temporal and spatial scales on model operation and processing. Design tools that can explore and mitigate the impacts of changing scales on linked model systems.

\section{Final Remarks}

For BGS the crucial issues to be addressed in the coming few years to see the successful deployment of an Environmental Modelling Platform for addressing the major questions surrounding energy, and water resource security and sustainability include:

1. The successful deployment of 3D geological models in an acceptable, understandable product form, from a dynamically constructed 3D geological framework model, that contains the heart of BGS geological information

2. The parameterisation of this framework model with physical properties and later chemical properties, with error and uncertainty bounds defined for line-work, lithostratigraphy and properties.

3. The use of this Environmental Modelling Platform with partners to provide the knowledge base for modelling Earth System processes at all scales. This requires linkages to climate models, surface process models, hydrological and hydraulic models and so on. But to achieve impact and value for society, coupling to social, economic and financial processes and models will also be necessary.

\section{Acknowledgments}

This paper is published with the permission of the Executive Director, British Geological Survey (NERC). The contribution of the reviewers are gratefully acknowledged: one anonymous reviewer and Pierre Glynn, USGS. 


\section{REFERENCES}

Berners-Lee T.,Hendler J, and Lassila O. The Sematic Web: A new form of Web content that is meaningful to computers will unleash a revolution of new possibilities. Scientific American http://www.cs.umd.edu/ golbeck/LBSC690/SemanticWeb.html

Chadwick, A. and Morfett, J., 1986. Hydraulics in Civil Engineering. Allen and Unwin (Publishers) Ltd, London, UK.

Giles, J.R.A.; Hughes, A.; Kessler, H.; Watson, C.; Peach, D.. 2010 Data, and research for applications and models (DREAM) : scoping study report. Nottingham, UK, British Geological Survey, 75pp. (OR/10/020) (Unpublished)

Glynn, P.D., 2016. Integrated environmental Modelling: human decisions, human challenges. In: Riddick, A.T., Kessler, H., Giles, J.R.A. (Eds.), Integrated Environmental Modelling to Solve Real World Problems: Methods, Vision and Challenges.

The Geological Society of London. http://dx.doi.org/10.1144/SP408.9.

Gregersen, J B; Gijsbers, P J A; Westen, S J P and Blind, M. 2005. OpenMI: The Essential Concepts and their Implications for Legacy Software. Advances in Geosciences, 4, 37-44, 2005.

Gregersen, J B; Gijsbers, P J A and Westen, S J P. 2007. OpenMI : Open Modelling Interface. Journal of Hydroinformatics, 9 (3), 175-191.

Harpham Q, Cleverly P and Kelly D, 2014. The FluidEarth 2 Implementation of OpenMI 2.0. Jnl of Hydroinformatics. Vol 16 No 4 pp 890-906.

Hughes, A.G.; Mansour, M.M.; Robins, N.S.. 2008 Evaluation of distributed recharge in an upland semi-arid karst system : the West Bank Mountain Aquifer, Middle East. Hydrogeology Journal, 16 (5). 845-854. 10.1007/s10040-008-0273-6

ISO Standard ISO 19115-1:2014

http://www.iso.org/iso/home/store/catalogue _ics/catalogue detail_ics.htm?csnumber=53798

Jackson C.R.; Spink A.E.F., 2004. User's manual for the groundwater flow model ZOOMQ3D. Nottingham, UK, British Geological Survey, 107pp. (IR/04/140) - http://nora.nerc.ac.uk/11829/

Mansour, Majdi; Mackay, Jonathan; Abesser, Corinna; Williams, Ann; Wang, Lei; Bricker, Stephanie; Jackson, Christopher. 2013 Integrated Environmental Modeling applied at the basin scale: linking different types of models using the OpenMI standard to improve simulation of groundwater processes in the Thames Basin, UK. In: MODFLOW and More 2013: Translating Science into Practice, Colorado, USA, 2-5 June 2013. (Unpublished)

Mansour, M., Hughes, A., O Dochartaigh, B., \& Graham, M. (2008). Representation of Urban Recharge Processes in the Distributed Recharge Model (ZOODRM) of the Glasgow urban area, Scotland. MODFLOW and More 2006: Managing Ground-Water Systems-Conference Proceedings, Poeter, Hill, \& Zheng..

Mansour, M.M.; Hughes, A.G.. 2004 User's manual for the distributed recharge model ZOODRM. British Geological Survey, 61pp. (IR/04/150) (Unpublished)

Merritt, J., Monaghan, A., Entwisle, D., Hughes, A., Campbell, D., \& Browne, M. (2007). 3D attributed models for addressing environmental and engineering geoscience problems in areas of urban regeneration-a case study in Glasgow, UK. first break, 25(8). 
Open Geospatial Consortium (OGC) 2013, Open Modelling Interface Standard (OpenMI), http://www.opengeospatial.org/standards/openmi

Royse, K.R.; Hughes, A.G., eds. 2014 Meeting report : NERC Integrated Environmental Modelling Workshop : held at the British Geological Survey, Keyworth, 4-5th

February. Nottingham, UK, British Geological Survey, 29pp. (OR/14/042) (Unpublished)

Sen, M.;Duffy, T. 2005 GeoSciML: Development of a generic GeoScience Markup Language. Computers and Geosciences, Volume 31, Issue 9

Smith, I.F. (Editor). 2005. Digital Geoscience Spatial Model Project Final Report. British Geological Survey Occaisional Publication No 9. 56pp

Turner, R.J.; Mansour, M.M.; Dearden, R.; O Dochartaigh, B.E.; Hughes, A.G.. 2015 Improved understanding of groundwater flow in complex superficial deposits using three-dimensional geological-framework and groundwater models: an example from Glasgow, Scotland (UK). Hydrogeology Journal, 23 (3). 493-506. 10.1007/s10040-014-1207-0

Voinov, A.; Kolagni, N;McAll, M.K.; Glynn, P.D.;Kragt M.E.; Ostermann, F.O;Pierce, A; Ramu, P. 2016, Modelling with stakeholders - Next Generation, Environmental Modelling and Software $77,1-25$ 


\section{Figure Descriptions:}

Fig. 1 Relationships between modelling concepts

Fig. 2. The structure of a linkable model component within the OpenMI framework

Fig. 3. Mechanism of passing data at run-time between linkable model components in OpenMI

Fig. 4. River Thames Catchment boundary and location and extent of models within the TIM model composition

Fig. 5. Model linkages and input/output parameters within the TIM model composition

Fig. 6. Modelling process in Glasgow 